\title{
Comparison of Lipid Profile, Liver Enzymes, Creatine Kinase and Lactate Dehydrogenase Among Type II Diabetes Mellitus Patients on Statin Therapy
}

This article was published in the following Dove Press journal: Diabetes, Metabolic Syndrome and Obesity: Targets and Therapy

\author{
Mezgebu Legesse Habte' \\ Daniel Seifu Melka ${ }^{2}$ \\ Maria Degef $\left(\mathbb{D}^{2}\right.$ \\ MKC Menon ${ }^{2}$ \\ Helen Yifter $^{3}$ \\ Teka Obsa Feyisa'
}

'Department of Biochemistry, School of Medicine, College of Health and Medical Sciences, Harmaya University, Harar, Ethiopia; ${ }^{2}$ Department of Medical Biochemistry, School of Medicine, College of Health Sciences, Addis Ababa University, Addis Ababa, Ethiopia; ${ }^{3}$ Department of Medical Endocrinology, School of Medicine, College of Health Sciences, Addis Ababa University, Addis Ababa, Ethiopia
Correspondence: Mezgebu Legesse Habte

Department of Biochemistry, School of Medicine, College of Health and Medical Sciences, Harmaya University, Ethiopia Tel +25I 934095576

Email mezgebulegesse@gmail.com
Background: Diabetes mellitus (DM) is an epidemic disease affecting millions worldwide; the majority being type 2 diabetes mellitus (T2DM). Diabetes mellitus has been shown to be an important risk factor for the development of a variety of cardiovascular diseases, which are becoming common in Ethiopia. Consequently, risk-reducing statin therapy is recommended for nearly all patients with T2DM at 40 years of age or older regardless of cholesterol level. However, some controversies exist regarding its safety.

Objective: The aim of this study was to assess and compare the levels of lipid profile, liver enzymes, creatine kinase-MB (CK-MB) and lactate dehydrogenase (LDH) among T2DM patients on statin therapy.

Methodology: A hospital-based cross-sectional study was conducted on a total of 100 T2DM patients. The study participants were divided into four groups consisting of equal numbers of participants $(\mathrm{n}=25)$. Group I, II, and III were T2DM patients who were on statin therapy for 14 days -6 months, 6-18 months and $>18$ months, respectively. Group IV consisted of T2DM patients who were not on statin therapy. Convenient sampling technique was implemented till the required number had been achieved. Sociodemographic data was collected by using a standardized questionnaire. Fasting blood was collected and lipid profile, liver enzymes, CK-MB, LDH and fasting blood sugar were analyzed. Data was entered using epi-data and analyzed by one way ANOVA followed by Tukey post hoc multiple comparison tests using SPSS V. 20.00. A $P$-value $<$ 0.05 was considered statistically significant.

Results: The mean values of total cholesterol and TAG were significantly lower among group III as compared to group I ( $P$-values $=0.019 \& 0.01)$. Similarly, LDL-c was significantly lower among group III as compared to group I $(P=0.022)$ and group IV $(P=0.027)$. Serum liver enzymes, CK-MB and LDH were not significantly different among the study groups $(P>0.05)$. The mean values of alanine aminotransferase (ALT) and AST were found within normal range while mean ALP was higher in all study groups. Fasting blood glucose value was not significantly different among the study groups, but higher than normal cut-off value in all groups.

Conclusion: Statin therapy taken for a longer time has an effect in lowering total cholesterol, LDL- $\mathrm{c}$ and TAG in T2DM patients. Statin therapy has not brought significant change on CK-MB, LDH, liver enzymes and other parameters among T2DM patients.

Keywords: CK-MB, HMG-COA reductase, lipid profile, liver enzymes, statin, T2DM, LDH

\section{Introduction}

Diabetes is a serious chronic disease that occurs either when the pancreas does not produce enough insulin or when the body cannot effectively use the insulin it produces. It is characterized by hyperglycemia with disturbances of carbohydrates, 
fat and protein metabolism. ${ }^{1}$ In Ethiopia, occurrences and complications of DM have been increasing over time at an alarming rate. ${ }^{2}$ The starting point for living well with diabetes is an early diagnosis followed by glycemic control. $^{3}$ One of the consequences of metabolic syndrome as a result of diabetes is dyslipidemia, which is characterized by elevated fasting and postprandial triglycerides (TG), low level of high density lipoprotein cholesterol (HDL-C), elevated level of low density lipoprotein- cholesterol (LDL-C) and the predominance of small dense LDL-C particles. Several studies have indicated that diabetic dyslipidemia is caused by impaired action of lipoprotein lipase (LPL) that is localized in the endothelial cells. Modified LDL such as glycated and oxidized LDL that play an important negative role to induce vascular and renal cellular dysfunction and cardiovascular risk which in turn hasten diabetic complications. ${ }^{4-6}$ Non-pharmacologic interventions (diet and exercise) are first line therapies and are adjuvant to the pharmacologic therapy when necessary in the treatment of diabetic dyslipidemia. Lowering LDL$\mathrm{C}$ level is the first priority in treating diabetic dyslipidemia mainly circulating levels of smaller and denser LDL particles.

High plasma level of LDL-C contributes to plaque formation in the arteries that narrows blood vessels and restrict blood flows. Under such conditions, statins are the first drug of choice, followed by resins, ezetimibe, fenofibrate, niacin and others. If a single agent is inadequate to achieve lipid goals, combinations of the drugs may be used. $^{7,8}$ Targets for LDL-C level are very important in choice of a pharmacological lipid-lowering agent. LDL-C $<1.8 \mathrm{mmol} / \mathrm{L}(70 \mathrm{mg} / \mathrm{dL})$ and TG $<2.3 \mathrm{mmol} / \mathrm{L}(204 \mathrm{mg} /$ dl) values are greatly desired for very high risk patients. ${ }^{9}$ Statin lowers atherogenic LDL-C through inhibition of 3hydroxy-3-methylglutaryl coenzyme-A (HMG-CoA) reductase, the rate-limiting enzyme of cholesterol biosynthesis and upregulation of LDL receptors on the cell membrane. Statin reduces CVD by $25-30 \% .{ }^{10}$ Statin has been introduced as the best cholesterol lowering drug since 1987 and it includes; atorvastatin, simvastatin, pravastatin and rosuvastatin. ${ }^{11}$ In addition, statin has anti-inflammatory, immunomodulation and anti-microbial effects. ${ }^{12,13}$ As a result, statins are considered very effective in reducing cardiovascular morbidity and mortality in high-risk patients like those with T2DM. ${ }^{14}$

Even if the safety of statins was well established, several clinical studies have demonstrated evidence of liver toxicity and statin-associated myopathy. Studies indicated that genetic and environmental factors determine statin-induced liver and muscle toxicity and its effectiveness as well. ${ }^{14-16}$ The other concern over statin safety is myopathy and rhabdomyolysis, which is characterized by increased activity of $\mathrm{CK}$ and LDH. ${ }^{16,17}$ There is no well documented study done to examine the extent of compliance and adherence to progression of liver function tests, serum CK and LDH level among T2DM patients receiving lipid-lowering drugs like statins in Ethiopia.

Higher doses of statins have been linked to an increased risk of a life-threatening form of muscle breakdown called rhabdomyolysis. This can lead to permanent kidney damage, coma, and death. Symptoms include fatigue, muscle pain, muscle tenderness, muscle weakness, nocturnal cramping and tendon pain. The mechanism of statin-induced myopathy could be impaired synthesis of cholesterol leading to changes in the cholesterol in myocyte membranes and behavior of the membrane, impaired synthesis of compounds in the cholesterol pathway (coenzyme Q10) and depletion of isoprenoids, which prevents myofibril apoptosis. ${ }^{18-20}$

Measuring serum CK is an important part of the evaluation of patients with muscle weakness or myalgia, and assessing of patients with myopathies or muscle injuries. ${ }^{21}$ Similarly, evaluation of serum $\mathrm{LDH}$ or $\mathrm{LDH}$ isoenzyme is relevant in the diagnosis, prognosis and monitoring of diseases such as myocardial infarction, hemolytic anemia, hepatocellular carcinoma, ovarian dysgerminoma and testicular germ cell tumor. ${ }^{22,23}$

Therefore, the present study was conducted to compare lipid profile, liver enzymes, creatine kinase and lactate dehydrogenase among type II diabetes mellitus patients on statin therapy at Tikur Anbessa Specialized Hospital (TASH) in Addis Ababa, Ethiopia.

\section{Materials and Methods}

\section{Study Area and Period}

This study was conducted at the Diabetic Clinic of Tikur Anbessa Specialized Hospital (TASH), Ethiopia from March, 2017 to September, 2017.

\section{Study Design}

A hospital-based cross-sectional study design was used to determine serum level of liver enzymes (alanine aminotransferase, ALT; AST and ALP), CK-MB isotype, LDH and lipid profiles (LDL, HDL, TAG and total cholesterol) among T2DM patients who were receiving statin and not receiving statin at the Diabetic Clinic of TASH 
within the study period. Since the study aimed at assessing the effect of statin drugs on lipid profile, liver enzymes, creatine kinase-MB (CK-MB) and lactate dehydrogenase (LDH) among T2DM patients with respect to duration of therapy, the study participants were grouped into four groups. Group I, II, and III were T2DM patients who were on statins for 14 days -6 months, 6-18 months and $>18$ months respectively. Group IV consisted of T2DM patients who were not on statin therapy. The number of study participants in each group was 25. Blood analysis was conducted at Zewditu Memorial Hospital, Ethiopia and at the Ethiopian Public Health Institution clinical chemistry laboratory unit.

\section{Inclusion and Exclusion Criteria}

T2DM patients who were on statin drugs, and T2DM patients who were not on statin drugs as a control group were included in this study. Patients who had any clinical evidence of cirrhosis or other causes of chronic liver disease, diagnosed T1DM, pregnancy, lactating mother, children less than 18 years of age, patients using HIV drugs and patients with known muscular and cardiac disease were excluded from this study.

\section{Data Collection}

\section{Body Mass Index (BMI) and Waist-to-Hip Ratio (WHR)}

Body mass index is a useful clinical calculation to diagnose obesity because it is correlated with total body fat. Values of BMI can be classified as follows. BMI $\leq 18.5$ Underweight, $\mathrm{BMI}=18.5-24$ normal weight, $\mathrm{BMI}=25-29.9$ overweight and BMI $\geq 30$ obese. BMI has been calculated as weight $(\mathrm{kg})$ divided by height in square meters e $\left(\mathrm{m}^{2}\right)$. Waistto-hip ratio to measure obesity which in turn is a possible indicator of other more serious health problems. According to WHO, WHR of above 0.9 for male and 0.8 for female is considered as abdominal obesity. Weight, height, waist and hip circumferences were measured by an experienced nurse and BMI and WHR were calculated.

\section{Blood Sample Collection}

A total of $5 \mathrm{~mL}$ of venous blood was drawn from each T2DM patient using a disposable plastic syringe. The blood was poured into a serum separator tube (SST) and then centrifuged after it was clotted. The serum was kept at $-80^{\circ} \mathrm{C}$ in a refrigerator till biochemical analysis was carried out. Liver enzymes (AST, ALT, ALP), CK-MB, LDH and lipid profile (LDL, HDL, TAG and total cholesterol) were measured with a spectrophotometer Cobas c analyzer. Manufacturer instructions of reagent and instrument were strictly followed. ${ }^{24}$

\section{Data Analysis}

The questionnaires were checked for completeness. Collected quantitative data was coded, entered into a computer, processed, edited, and analyzed using EPI-INFO and SPSS (version 20). One-way analysis of variance (ANOVA) was done to determine statistical differences among all groups of the study participants. Pairwise comparisons were conducted by Tukey post hoc multiple comparison tests. T-test also used to compare data between groups. The results of the data were presented as mean \pm standard deviation (SD). A $P$-value $<0.05$ was considered statistically significant.

\section{Ethical Considerations}

This study was conducted in accordance with the declaration of Helsinki Oct 2008 (DoH/Oct2008). The ethical clearance was obtained from the Department of Biochemistry Research and Ethical Review Committee (DRERC), by approval letter with Ref. No. of SOM/BCHM/151/2009, meeting No. of DRERC 01/17 and protocol No. of M.Sc. 8/17 issued on March 09/2017. A consent form (Figure 1), prepared with detailed explanation of objectives, risks and benefits of the study was presented to the participants and the confidentiality was strictly kept. Data was collected after obtaining informed consent and agreement from the patients under study. Sample collection was performed by trained health professionals following ethical steps and scientific procedures.

\section{Results}

Among the total participants, 48/100 (48\%) were males and $52 / 100(52 \%)$ were females and the numbers of male and female in each group were more or less similar. The mean age of the participants in groups I, II, III and IV was $57 \pm 7.77,55.28 \pm 7.51,58.4 \pm 9.36$ and $52.9 \pm 8.46$ years, respectively. The total (ground) mean age was $55.92 \pm$ 8.69 years, ranging between 38 and 74 years. About 89/ $100(89 \%)$ of the study participants had been educated up to preparatory and above and 11/100 (11\%) were illiterate. None of the study participants were on the highest dose (80 mg) of statin therapy. About 40/75 (53.3\%) and 35/75 (46.7\%) of the study participants used $20 \mathrm{mg}$ and $40 \mathrm{mg}$ statin drugs respectively.

Of the total participants in this study, simvastatin, atorvastatin, lovastatin and fluvastatin were used by $29 /$ 


\section{CONSENT FORM}

In undersigning the objective of this study, entitled, "comparison of liver enzymes, creatine kinase and lactate dehydrognase at different period of T2DM patients who are on statin attending diabetic clinic of Tikur Anbesa Specialized Hospital Addis Ababa, Ethiopia”, I am giving my consent to participate in the study. I have been informed that the purpose of this study is to assess serum level of liver enzymes, creatine kinase and lactate dehydrognase and associated risk factors at different period of T2DM patients who are on statin attending diabetic clinic of Tikur Anbesa Specialized Hospital Addis Ababa, Ethiopia, I have understood that participation in this study is entirely voluntarily. I have been told that my answers to the questions will not be given to anyone else and no reports of this study ever identify me in any way. I have also been informed that my participation or non-participation or my refusal to answer questions will have no effect on me. I understood that participation in this study does not involve risks. I understood that Mezgebu legesse is the contact person if I have questions about the study or about my rights as a study participant.

Respondent's signature

Interviewer

Name __ Signature ___ Date

Figure I Study consent form.

75 (38.67\%), 28/75 (37.33\%), 15/75 (20\%) and 3/75 (4\%) respectively as anti-dyslipidemia drugs (Figure 2).

This study indicated that metformin was the most frequently prescribed $(44 / 100,44 \%)$ anti- glycemic drug followed by insulin $(19 / 100,19 \%)$ among the study participants. And around 37/100 (37\%) of the participants were on a combination of anti-glycemic drugs (Figure 3).

The mean SBP, DBP, BMI, and WHR were not significantly different among the four study groups. One way ANOVA indicated that mean SBP in T2DM patients who were on statin drugs for $>18$ months was higher (142 $\mathrm{mmHg}$ ) as compared with other study groups. Groups of study participants who were on statins for 14 days6 months and who were not on statin therapy had similar mean SBP. The mean DBP was found to be in normal range among all study groups. The mean values of

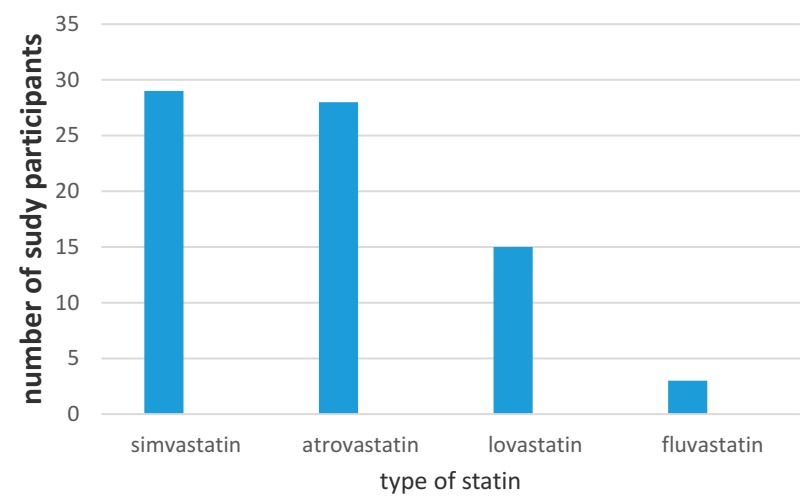

Figure 2 Frequency of statin type used by study participants.
BMI and WHR were higher than normal reference ranges in all study groups (Table 1).

The mean values of LDL, TAG, and total cholesterol showed statistically significant differences among the four study groups as determined by one way ANOVA. Post hoc Tukey's test revealed that total cholesterol and TAG were significantly lower in group III than in group I $(\mathrm{P}=0.019$ $\& 0.01)$, LDL was significantly lower in group III than in group I $(\mathrm{P}=0.022)$ and group IV $(\mathrm{P}=0.027)$. But, HDLcholesterol and fasting blood glucose did not indicate a statistically significant difference among the study groups (Table 2).

In this study, the mean level of serum liver enzymes (ALT, AST and ALP) showed slight elevation among T2DM patients who were on statin therapy for greater than 18 months as compared to other study groups but,

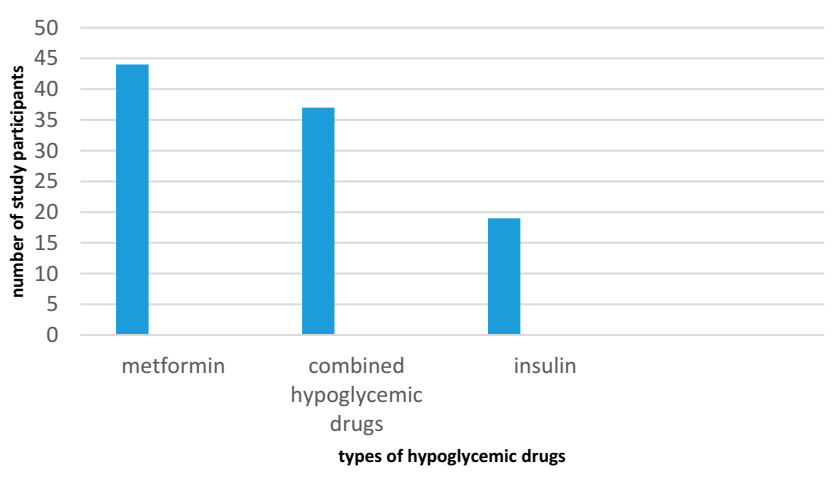

Figure 3 Type of hypoglycemic drugs used by study participants. 
Table I Anthropometric and Clinical Characteristics of T2DM Patients Who Were on Statin Drugs

\begin{tabular}{|c|c|c|c|}
\hline Variable & Groups & Mean \pm SD & $P$-value \\
\hline SBP & $\begin{array}{l}14 \text { days }-6 \text { months }(n=25) \\
6 \text { months }-18 \text { months }(n=25) \\
>18 \text { months }(n=25) \\
\text { Not on statin therapy }(n=25) \\
\text { Total }(N=100)\end{array}$ & $\begin{array}{l}136.40 \pm 14.40 \\
133.44 \pm 15.19 \\
142.00 \pm 20.62 \\
136.80 \pm 21.55 \\
137.16 \pm 18.20\end{array}$ & 0.418 \\
\hline DBP & $\begin{array}{l}14 \text { days }-6 \text { months }(n=25) \\
6 \text { months }-18 \text { months }(n=25) \\
>18 \text { months }(n=25) \\
\text { Not on statin therapy }(n=25) \\
\text { Total }(N=100)\end{array}$ & $\begin{array}{l}80.40 \pm 6.76 \\
79.36 \pm 9.59 \\
82.76 \pm 9.33 \\
82.80 \pm 12.08 \\
81.33 \pm 9.60\end{array}$ & 0.494 \\
\hline BMI & $\begin{array}{l}14 \text { days }-6 \text { months }(n=25) \\
6 \text { months }-18 \text { months }(n=25) \\
>18 \text { months }(n=25) \\
\text { Not on statin therapy }(n=25) \\
\text { Total }(N=100)\end{array}$ & $\begin{array}{l}26.43 \pm 3.10 \\
26.69 \pm 3.73 \\
28.99 \pm 5.89 \\
26.00 \pm 2.99 \\
27.03 \pm 4.20\end{array}$ & 0.052 \\
\hline WHR & $\begin{array}{l}14 \text { days }-6 \text { months }(n=25) \\
6 \text { months }-18 \text { months }(n=25) \\
>18 \text { months }(n=25) \\
\text { Not on statin therapy }(n=25) \\
\text { Total }(N=100)\end{array}$ & $\begin{array}{l}0.96 \pm 0.10 \\
0.95 \pm 0.06 \\
0.97 \pm 0.10 \\
0.97 \pm 0.06 \\
0.96 \pm 0.07\end{array}$ & 0.754 \\
\hline
\end{tabular}

Abbreviations: SBP, Systolic blood pressure; DBP, Diastolic blood pressure; BMI, Body mass index; WHR, Waist-hip-ratio.

statistically not significant. The mean values of both serum ALT and AST in all study groups were found within normal reference ranges (Tables 3 and 4).

One way ANOVA showed serum level of CK-MB isotype was higher among T2DM patients who were on statin therapy for greater than 18 months as compared to other study groups, but not statistically significant. For all study groups, serum mean value of CK-MB isotype was found within the normal reference range $(\mathrm{CK}-\mathrm{MB}<25 \mathrm{U} /$ L). The mean value of LDH was higher among T2DM patients who were on statin for 14 days 6 months and greater than 18 months, but statistically not significant. For all study groups mean values of LDH were found within the normal reference range (Table 4), but it was pulled to the upper limit (Table 5).

There was an elevation of serum total cholesterol and LDL among T2DM patients who were not on statin therapy as compared to T2DM patients who were on statin therapy. The mean values of liver enzymes (ALT, AST, and ALP), CK-MB isotype and LDH were slightly higher among T2DM patients who were on statin therapy than T2DM patients who were not on statin therapy but, statistically not significant. HDL-cholesterol was
Table 2 Serum Lipid Profile and Glucose Levels in T2DM Patients Who Were on Statin Therapy

\begin{tabular}{|c|c|c|c|}
\hline Variables & Groups & $\begin{array}{l}\text { Mean } \pm \text { SD }(\mathrm{mg} / \\
\mathrm{dL})\end{array}$ & P-value \\
\hline TC & $\begin{array}{l}14 \text { days }-6 \text { months }(n=25) \\
6 \text { months }-18 \text { months } \\
(n=25) \\
>18 \text { months }(n=25) \\
\text { Not on statin therapy } \\
(n=25) \\
\text { Total }(N=100)\end{array}$ & $\begin{array}{l}216.04 \pm 59.36^{\mathrm{a}} \\
186.16 \pm 50.89 \\
172.48 \pm 46.57^{\mathrm{b}} \\
202.56 \pm 49.55 \\
194.31 \pm 53.62\end{array}$ & 0.022 \\
\hline HDL-C & $\begin{array}{l}14 \text { days }-6 \text { months }(n=25) \\
6 \text { months- } 18 \text { months } \\
(n=25) \\
>18 \text { months }(n=25) \\
\text { Not on statin therapy } \\
(n=25) \\
\text { Total }(n=100)\end{array}$ & $\begin{array}{l}34.60 \pm 9.60 \\
32.56 \pm 10.36 \\
35.28 \pm 13.749 \\
34.64 \pm 8.12 \\
34.27 \pm 10.547\end{array}$ & 0.820 \\
\hline LDL-C & $\begin{array}{l}14 \text { days }-6 \text { months }(n=25) \\
6 \text { months }-18 \text { months } \\
(n=25) \\
>18 \text { months }(n=25) \\
\text { Not on statin therapy } \\
(n=25) \\
\text { Total }(N=100)\end{array}$ & $\begin{array}{l}139.88 \pm 41.75^{\mathrm{a}} \\
118.48 \pm 38.90 \\
107.60 \pm 35.364^{\mathrm{c}} \\
139.16 \pm\left. 40.2\right|^{\mathrm{d}} \\
126.28 \pm 40.94\end{array}$ & 0.008 \\
\hline TAG & $\begin{array}{l}14 \text { days }-6 \text { months }(n=25) \\
6 \text { months }-18 \text { months } \\
(n=25) \\
>18 \text { months }(n=25) \\
\text { Not on statin therapy } \\
(n=25) \\
\text { Total }(N=100)\end{array}$ & $\begin{array}{l}233.84 \pm 108.146^{\mathrm{a}} \\
191.64 \pm 89.75 \\
148.24 \pm 69.87^{\mathrm{b}} \\
177.56 \pm 107.29 \\
187.82 \pm 98.61\end{array}$ & 0.018 \\
\hline FBG & $\begin{array}{l}14 \text { days }-6 \text { months }(n=25) \\
6 \text { months- } 18 \text { months } \\
(n=25) \\
>18 \text { months }(n=25) \\
\text { Not on statin therapy } \\
(n=25) \\
\text { Total }(N=100)\end{array}$ & $\begin{array}{l}167.24 \pm 53.44 \\
164.80 \pm 48.87 \\
169.60 \pm 76.79 \\
185.60 \pm 73.98 \\
171.81 \pm 63.99\end{array}$ & 0.662 \\
\hline
\end{tabular}

Notes: andicates significant differences compared to group III; ${ }^{b}$ Indicates significant differences compared to group I; Indicates significant differences compared to group I\&IV; Indicates significant differences compared to group III at $P<0.05$ as tested by Tukey post hoc multiple comparisons.

Abbreviations: TAG, Triacylglycerol; LDL, Low density lipoprotein; HDL, High density lipoprotein; TC, Total cholesterol; FBG, Fasting Blood glucose.

more or less similar among T2DM patients who were on statin and those had not yet started on statin drugs (Table 6).

The mean values of total cholesterol, LDL and TAG were higher among T2DM patients who were on $20 \mathrm{mg}$ statin therapy as compared to T2DM patients who were on $40 \mathrm{mg}$ statin therapy but, statistically not significant. The mean values of liver enzymes (ALT, AST, and ALP), CK-BM 
Table 3 Comparison of Liver Enzymes (ALT, AST and ALP) in T2DM Patients Who Were on Statin Therapy

\begin{tabular}{|c|c|c|c|}
\hline Variable & Groups & Mean \pm SD (IU/L) & $P$-value \\
\hline ALT & $\begin{array}{l}14 \text { days }-6 \text { months }(n=25) \\
6 \text { months }-18 \text { months }(n=25) \\
>18 \text { months }(n=25) \\
\text { Not on statin therapy }(n=25) \\
\text { Total }(N=100)\end{array}$ & $\begin{array}{l}23.08 \pm 14.1 \\
19.88 \pm 6.8 \\
23.92 \pm 8.9 \\
21.2 \pm 10.4 \\
22.2 \pm 10.3\end{array}$ & 0.452 \\
\hline AST & $\begin{array}{l}14 \text { days }-6 \text { months }(n=25) \\
6 \text { months }-18 \text { months }(n=25) \\
>18 \text { months }(n=25) \\
\text { Not start statin }(n=25) \\
\text { Total }(N=100)\end{array}$ & $\begin{array}{l}20.2 \pm 7.9 \\
19.6 \pm 4.9 \\
22.6 \pm 6.9 \\
19.4 \pm 10.7 \\
20.3 \pm 7.9\end{array}$ & 0.638 \\
\hline ALP & $\begin{array}{l}14 \text { days }-6 \text { months }(n=25) \\
6 \text { months }-18 \text { months }(n=25) \\
>18 \text { months }(n=25) \\
\text { Not start statin }(n=25) \\
\text { Total }(N=100)\end{array}$ & $\begin{array}{l}195.7 \pm 45.4 \\
187.2 \pm 57.2 \\
215.7 \pm 74.3 \\
184.6 \pm 36 \\
195.6 \pm 55.7\end{array}$ & 0.189 \\
\hline
\end{tabular}

Abbreviations: ALT, Alanine aminotransferase; AST, Aspartate aminotransferase; ALP, Alkaline phosphatase; $n$, Numbers participants in each groups; $N$, Total study subjects.

Table 4 Reference Cut- off- Value for Biochemical Parameters Performed in the study. Reference Range for Lipid Profiles

\begin{tabular}{|l|l|l|l|}
\hline Parameters & Desirable & Borderline & High \\
\hline TC & $<200 \mathrm{mg} / \mathrm{dL}$ & $200-239 \mathrm{mg} / \mathrm{Dl}$ & $>240 \mathrm{mg} / \mathrm{dL}$ \\
TG & $<150 \mathrm{mg} / \mathrm{dL}$ & $150-199 \mathrm{mg} / \mathrm{dL}$ & $>200 \mathrm{mg} / \mathrm{dL}$ \\
LDL-C & $<129 \mathrm{mg} / \mathrm{dL}$ & & $>160 \mathrm{mg} / \mathrm{DI}$ \\
HDL-C & $\geq 60 \mathrm{mg} / \mathrm{dL}$ & & \\
\hline
\end{tabular}

Table 5 Comparison of LDH and CK-MB Isotype Among T2DM Patients Who Were on Statin Drugs

\begin{tabular}{|l|l|l|l|}
\hline Variable & Groups & Mean \pm SD (IU/L) & P-value \\
\hline \multirow{2}{*}{ LDH } & 14 days-6 months $(n=25)$ & $191.6 \pm 77.9$ & 0.270 \\
& 6 months-18 months $(n=25)$ & $174.3 \pm 38.7$ & \\
& $>18$ months $(n=25)$ & $191.7 \pm 32.3$ & \\
& Not start statin $(n=25)$ & $168.8 \pm 44.1$ & \\
& Total $(N=100)$ & $181.6 \pm 55.7$ & \\
\hline \multirow{2}{*}{ CK-MB } & 14 days-6 months $(n=25)$ & $13.6 \pm 13.0$ & \\
& 6 months-18 months $(n=25)$ & $15.3 \pm 5.3$ & \\
& $>18$ months $(n=25)$ & $17.01 \pm 8.0$ & \\
& Not start statin $(n=25)$ & $14.7 \pm 10$ & \\
& Total $(N=100)$ & $15.2 \pm 9.7$ & \\
& &
\end{tabular}

Abbreviations: LDH, Lactate dehydrogenase; CK-MB isotype, Creatine kinase of heart muscle isotype; $n$, Numbers of study participants in the study groups; $N$, Total numbers of study participants.

isotype and LDH were slightly lower among T2DM patients who were on $20 \mathrm{mg}$ statin therapy than T2DM patients who were on $40 \mathrm{mg}$ statin therapy but statistically they were not significant (Table 7).
Table 6 Comparison of Biochemical Parameters Among T2DM Who Were on Statin and T2DM Who Were Not on Statin Therapy

\begin{tabular}{|l|l|l|l|}
\hline $\begin{array}{l}\text { Parameter } \\
\text { Mean } \pm \text { SD) }\end{array}$ & $\begin{array}{l}\text { T2DM Who } \\
\text { Were on Statin } \\
(\mathbf{n}=75)\end{array}$ & $\begin{array}{l}\text { T2DM Who Were } \\
\text { Not on Statin } \\
(\mathbf{n}=\mathbf{2 5})\end{array}$ & P-value \\
\hline TC (mg/dL) & $192 \pm 54.9$ & $203 \pm 49.5$ & 0.377 \\
LDL-C (mg/dL) & $122 \pm 40.5$ & $139 \pm 40.2$ & 0.069 \\
HDL-C (mg/dL) & $34.15 \pm 11.3$ & $34.64 \pm 8.2$ & 0.841 \\
TAG (mg/dL) & $177 \pm 96$ & $191 \pm 107$ & 0.551 \\
FBG (mg/dL) & $167 \pm 60$ & $185 \pm 73.9$ & 0.215 \\
ALT (IU/L) & $22.49 \pm 10.4$ & $21.2 \pm 10.6$ & 0.593 \\
AST (IU/L) & $20.6 \pm 6.7$ & $19.5 \pm 10.7$ & 0.540 \\
ALP (IU/L) & $199.4 \pm 60.5$ & $184.2 \pm 36.4$ & 0.237 \\
CK-MB (IU/L) & $15.3 \pm 9.4$ & $13.7 \pm 10.9$ & 0.587 \\
LDH (IU/L) & $185.88 \pm 53.5$ & $168.7 \pm 44.1$ & 0.152 \\
\hline
\end{tabular}

Abbreviations: ALP, Alkaline phosphatase; ALT, Alanine aminotransferase; AST, Aspartate aminotransferase; BD, Blood glucose; CK-MB, Creatine kinase heart isotype; HDL-C, High density cholesterol; LDH, Lactate dehydrogenase; LDL-C, Low density lipoprotein cholesterol; SD, Standard deviation; TAG, Triacylglycerol; TC, Total cholesterol.

Table 7 Comparison of Biochemical Parameters Among T2DM Patients Who Were on $20 \mathrm{mg}$ Statin Therapy and T2DM Patients Who Were on $40 \mathrm{mg}$ Statin Therapy

\begin{tabular}{|c|c|c|c|}
\hline $\begin{array}{l}\text { Parameter } \\
\text { (Mean } \pm \text { SD) }\end{array}$ & $\begin{array}{l}\text { T2DM Who } \\
\text { Were on } 20 \mathrm{mg} \\
\text { Statin }(n=40)\end{array}$ & $\begin{array}{l}\text { T2DM Who } \\
\text { Were on } 40 \text { mg } \\
\text { Statin }(n=35)\end{array}$ & P-value \\
\hline TC (mg/dL) & $203.6 \pm 57$ & $|8| \pm 5 \mid$ & 0.076 \\
\hline LDL-C (mg/dL) & $|3| \pm 4 \mid$ & $114 \pm 38$ & 0.071 \\
\hline $\mathrm{HDL}-\mathrm{C}(\mathrm{mg} / \mathrm{dL})$ & $33.4 \pm 11.6$ & $35 \pm 11$ & 0.530 \\
\hline TAG (mg/dL) & $209.6 \pm 112$ & $175 \pm 76$ & 0.122 \\
\hline FBG (mg/dL) & $174 \pm 46$ & $160.5 \pm 70$ & 0.308 \\
\hline ALT (IU/L) & $20.68 \pm 11.0$ & $24.57 \pm 9.4$ & 0.107 \\
\hline AST (IU/L) & $20.0 \pm 7.3$ & $20.94 \pm 5.9$ & 0.682 \\
\hline ALP (IU/L) & $196.7 \pm 37.5$ & $202.2 \pm 75.5$ & 0.670 \\
\hline CK-MB (IU/L) & $14.4 \pm 6.3$ & $15.7 \pm \mid 1.4$ & 0.654 \\
\hline LDH (IU/L) & $175.2 \pm 34$ & $195.2 \pm 64$ & 0.106 \\
\hline
\end{tabular}

Abbreviations: ALP, Alkaline phosphatase; ALT, Alanine aminotransferase; AST, Aspartate aminotransferase; BD, Blood glucose; CK-MB, Creatine kinase heart isotype; HDL-C, High density cholesterol; LDH, Lactate dehydrogenase; LDL-C, Low density lipoprotein cholesterol; SD, Standard deviation; TAG, Triacylglycerol; TC, total cholesterol.

One way ANOVA showed that the mean values of serum total cholesterol and LDL cholesterol had statistically significant differences among T2DM patients who were on metformin + statin, T2DM patients who were on insulin + statin therapy and T2DM patients who were on combined hypoglycemic drugs + statin $(\mathrm{P}$-value $=0.034$ for total cholesterol and 0.046 for LDL-cholesterol). A Tukey Post hoc test showed that the mean values of serum total cholesterol and LDL cholesterol were significantly higher 
Table 8 Comparison of Lipid Profile, Blood Glucose, Liver Enzymes, CK-MB and LDH Among T2DM Patients Who Were on Metformin + Statin, Insulin + Statin and Combined Hypoglycemic Drugs + Statin Therapy

\begin{tabular}{|c|c|c|c|c|}
\hline \multirow{2}{*}{$\begin{array}{l}\text { Parameter } \\
\text { (Mean } \pm S D)\end{array}$} & \multicolumn{3}{|l|}{ Treatment } & \multirow[t]{2}{*}{ P-value } \\
\hline & $\begin{array}{l}\text { Metformin } \\
+ \text { Statin } \\
(n=34)\end{array}$ & $\begin{array}{l}\text { Insulin + } \\
\text { Statin } \\
(n=17)\end{array}$ & $\begin{array}{l}\text { Combined } \\
\text { Hypo- } \\
\text { glycemic + } \\
\text { Statin }(n=24)\end{array}$ & \\
\hline $\mathrm{TC}(\mathrm{mg} / \mathrm{dL})$ & $209 . \pm 54.5$ & $171.6 \pm 50$ & $191 \pm 54.9$ & $0.034 *$ \\
\hline $\begin{array}{l}\text { LDL-C } \\
(\mathrm{mg} / \mathrm{dL})\end{array}$ & $138 \pm 40$ & $110 \pm 36$ & $112 \pm 39.9$ & $0.046 *$ \\
\hline $\begin{array}{l}\text { HDL-C (mg/ } \\
\mathrm{dL})\end{array}$ & $29.9 \pm 12.8$ & $36 \pm 10.8$ & $33.2 \pm 10$ & 0.092 \\
\hline TAG $(\mathrm{mg} / \mathrm{dL})$ & $205.9 \pm 100$ & $|53.2 \pm 5|$ & $197.4 \pm 109$ & 0.169 \\
\hline FBG $(\mathrm{mg} / \mathrm{dL})$ & $174 \pm 63.4$ & $147 \pm 67$ & $170.6 \pm 52$ & 0.936 \\
\hline ALT (IU/L) & $22.18 \pm 10$ & $19.94 \pm 8$ & $24.75 \pm 12$ & 0.342 \\
\hline AST (IU/L) & $21.5 \pm 7.3$ & $|8.7| \pm 4.3$ & $20.3 \pm 7.1$ & 0.372 \\
\hline ALP (IU/L) & $208.3 \pm 67$ & $203.2 \pm 75$ & $|84.2 \pm 3|$ & 0.317 \\
\hline $\begin{array}{l}\text { CK-MB } \\
(\mathrm{IU} / \mathrm{L})\end{array}$ & $16.7 \pm 12$ & $14.2 \pm 7.3$ & $14.3 \pm 5.9$ & 0.508 \\
\hline LDH (IU/L) & $193 \pm 69.4$ & $190.88 \pm 34.2$ & $|7| \pm 33.6$ & 0.254 \\
\hline
\end{tabular}

Note: *Statistically significant difference.

Abbreviations: ALP, Alkaline phosphatase; ALT, Alanine aminotransferase; AST, Aspartate aminotransferase; BD, Blood glucose; CK-MB, Creatine kinase heart isotype; HDL-C, High density cholesterol; LDH, Lactate dehydrogenase; LDL-C, Low density lipoprotein cholesterol; SD, Standard deviation; TAG, Triacylglycerol; TC, Total cholesterol.

among T2DM patients who were on metformin + statin therapy than T2DM patients who were on insulin + statin therapy. The mean value of HDL cholesterol was higher in T2DM patients who were on insulin + statin therapy than in the other two groups. The mean values of serum glucose, liver enzymes (ALT, AST, and ALP), CK-MB isotype and LDH were not significantly different among the three groups (Table 8 ).

\section{Discussion}

Dyslipidemia is one of the most characterized metabolic syndromes as a result of diabetes, especially T2DM patients who are considered a high risk group for developing CVD. ${ }^{4,5}$ As a result, all T2DM patients above the age of 40 years are recommended to take anti-dyslipidemic drugs, mainly statins. ${ }^{25}$ Statins, HMG-CoA reductase inhibitors, are the most widely used medications for decreasing LDL cholesterol and play a vital role in the prevention of atherosclerotic cardiovascular complications. ${ }^{19}$ In the present study, simvastatin, atorvastatin and lovastatin were prescribed for 29 (38.7\%), $28(37.3 \%)$ and $15(20 \%)$ of the total 75 study participants, respectively.
Statin works by inhibiting HMG-CoA reductase, causing upregulation of the LDL-C receptors on the surface of the liver cell and increasing the removal of LDL-C from the blood. HMG-CoA reductase is a rate-limiting enzyme in cholesterol biosynthesis. In addition, statins lower serum triglycerides concentrate and modify endothelial function, inflammatory responses, plaque stability, and thrombus formation. As a result, statin appears to play a major role in decreasing the risk of coronary heart disease (CHD) and all-cause mortality. ${ }^{26,27}$

The results of the present study indicated that the mean serum values of total cholesterol, LDL- cholesterol, and TAG were significantly different among the four study groups (group I, II, III and IV). Post hoc Tukey's multiple comparison test revealed that total cholesterol and TAG concentration were significantly lowered in group III as compared to group I $(\mathrm{P}=0.019,0.01)$. In addition, $\mathrm{LDL}$ cholesterol was significantly lowered in group III compared to group I $(\mathrm{P}=0.022)$ and group IV $(\mathrm{P}=0.027)$ (Table 2). Similarly, mean serum level of LDL-cholesterol was lower in T2DM patients who were on statin therapy than in T2DM patients who were not on statin therapy ( $\mathrm{P}=0.069$ ) (Table 6). The result of this study was in line with studies done by Trentman et $\mathrm{al}^{26}$ and Stancu and $\mathrm{Sima}_{28}$ to assess the efficacy of statin drugs Other studies done to investigate the long term efficacy and safety of statin therapy by Takata et $\mathrm{al},{ }^{29}$ Ford et al, ${ }^{30}$ and Lu et al ${ }^{31}$ support this result.

The mean value of all serum lipid panels in all study groups were higher than the normal reference range, which may be due to T2DM. This is supported by a study done by Borle et al to assess dyslipidemia in T2DM patients which showed $86 \%$ had dyslipidemia. ${ }^{1}$ Independent $t$-test in this study indicated that mean values of serum total cholesterol, LDL cholesterol, and TAG were higher among T2DM patients who were on $20 \mathrm{mg}$ statin as compared to T2DM patients who were on $40 \mathrm{mg}$ statin therapy (P-values $=0.076$ for total cholesterol, 0.071 for LDL cholesterol, and 0.122 for TAG) (Table 7). This result agrees with a study done by Pedro-Botet et $\mathrm{al}^{19}$ that indicated high dose statin is necessary to achieve the desired plasma lipid goal. The mean values of serum total cholesterol and LDL cholesterol in this study were significantly different among T2DM patients who were on metformin + statin, insulin + statin and combined hypoglycemic drugs + statin therapy $(P=0.034$ for total cholesterol and $\mathrm{P}=0.046$ for LDL) (Table 8 ). These may be due to better glucose control potential and the multiple 
effect of insulin in the metabolism of glucose and fat by muscle and other tissue.

The anatomic and physiologic nature of liver makes it central to metabolism of virtually every foreign substance including most drugs like statin. ${ }^{32-34}$ Results of the present study showed that the mean values of serum liver enzymes (ALT, AST, and ALP) had not shown statistically significant elevation among T2DM patients who were on statins for longer than 18 months as compared to the other groups. Both mean serum ALT and AST were found to be within the normal reference range (Table 9) in all of the study groups (Table 3). However, some studies revealed increased levels of aminotransferases in patients who were on atorvastatin therapy for 8 months. ${ }^{35}$ Clinical trials done by Thapar et $\mathrm{al}^{36}$ have shown that statin therapy has been associated with elevations in serum ALT levels in approximately 3\% of persons who have received statin drugs. A study done in China by Gao et $\mathrm{al}^{37}$ also indicated that ALT $>1 \times \mathrm{ULN}$ was higher among statin users.

In the present study, absence of significant elevation in liver enzyme (ALT, AST and ALP) levels among statin users at different time periods may be due to the tolerability of statins by the study participants. Another reason may be due to the absence of high dose statin usage by the study participants (53.3\% and $46.7 \%$ of the study participant used $20 \mathrm{mg}$ and $40 \mathrm{mg}$ statin, respectively). In addition, none of the participants in this study was a chronic alcoholic, a cigarette smoker or took drugs other than hypoglycemic and statin drugs. All the above factors have an impact on liver function and exacerbate adverse effects of statins. The mean serum value of ALP was above the normal reference range () in all the study groups (Table 2). Elevation of mean serum ALP in all of the study groups may be due to dyslipidemia as all groups of study participants had higher mean lipid profile and poor blood glucose control (Tables 2 and 3). Liver enzymes

Table 9 Reference Cut-off-Value for Biochemical Parameters Performed in the study. Reference Ranges for Liver and Heart Muscle Enzymes

\begin{tabular}{|l|l|l|}
\hline Parameters & Normal Value & Higher \\
\hline ALT & $5-40 \mathrm{IU} / \mathrm{L}$ & $>40 \mathrm{IU} / \mathrm{L}$ \\
AST & $5-40 \mathrm{IU} / \mathrm{L}$ & $>40 \mathrm{IU} / \mathrm{L}$ \\
ALP & $35-130 \mathrm{IU} / \mathrm{L}$ & $>130 \mathrm{IU} / \mathrm{L}$ \\
$\mathrm{CK}-\mathrm{MB}$ & $5-25 \mathrm{IU} / \mathrm{L}$ & $>25 \mathrm{IU} / \mathrm{L}$ \\
LDH & $140-280 \mathrm{IU} / \mathrm{L}$ & $>280 \mathrm{IU} / \mathrm{L}$ \\
\hline
\end{tabular}

Abbreviations: ALP, Alkaline phosphatase; ALT, Alanine aminotransferase; AST, Aspartate aminotransferase; CK-MB, Creatine kinase heart isotype; LDH, Lactate dehydrogenase.
(ALT, AST and ALP) showed statistically non-significant elevation among T2DM patients who were on statin therapy as compared to T2DM patients who were not on statin therapy (Table 6). These elevations of liver enzymes could be due to statins targetting liver for its antidyslipidemic effect and metabolism and its interaction with hypoglycemic drugs; thereby inducing mild hepatotoxicity. ${ }^{28,38,39}$

Similarly, serum mean values of liver enzymes (ALT, AST and ALP) were higher among T2DM patients who were on $40 \mathrm{mg}$ statin therapy than T2DM patients who were on $20 \mathrm{mg}$ statin therapy (Table 7). These may be due to dose-dependent idiosyncratic drug-induced liver injury and its pathogenesis is complex and depends on different factors. ${ }^{40}$ The mean values of liver enzymes (ALT, AST and ALP) among T2DM patients who were on metformin + statin, insulin + statin and combined hypoglycemic drugs + statin were not significantly different (Table 8 ). This may be due to the smaller sample size, study design effect, and degree of tolerability of hypoglycemic drugs and statin and their interaction by the liver.

Statin can induce muscle cell injuries by impaired synthesis of coenzyme Q10 (ubiquinone) for mitochondrial function, depletion of isoprenoids and disruption of cell membrane integrity. ${ }^{18}$ Quantitative measurement of serum Creatine kinase-MB (CK-MB) isotype has been reported to be useful for the diagnosis of myocardial infarction, re-infarction, and the size of infarction. Creatine kinase-MB can also be used as a secondary marker to aid in the diagnosis and measurement of the degree of myocardial necrosis as a result of drug toxicity. ${ }^{41}$ Out of the total CK isotypes found in the serum, CK-MB accounts for $6-25 \%$. The finding of this study indicated that the mean serum level of CK-MB isotype was within normal reference range (Table 5) among T2DM patients who were on statin for greater than 18 months as compared to other study groups. This result is supported by a study done by Parker et $\mathrm{al}^{42}$ to measure serum CK before and after atorvastatin or placebo was administered for 6 months to 420 healthy, statinnaive subjects and indicated no individual $\mathrm{CK}$ value $\geq 10^{\times}$ ULN. Another study done by Soko et $\mathrm{al}^{43}$ in Zimbabwe did not show any elevation of serum CK. A study done by Calza et $\mathrm{al}^{44}$ showed that statin-induced elevation of CK is positively related with duration of statin use. A non-significant increase in CK-MB in our study may be due to the absence of high statin dose (80 mg), chronic alcoholism, cigarette smoking and drugs other than hypoglycemic and statin by the study participants.

The mean value of serum CK-MB isotype level among T2DM patients who were on statin therapy showed nonsignificant elevation as compared to T2DM patients who 
were not on statin therapy (Table 6). The cause for serum CK elevation could be quite diverse, including excessive muscular stress and ischemia, genetic defects, and direct toxicity or physical damage, drugs and alcohol. ${ }^{45}$ Statin drugs can disrupt the normal cardiac myocyte membrane integrity leading to release of intracellular components including structural proteins and enzymes such as CKMB isotype in serum. ${ }^{41}$ Similarly, CK-MB isotype among T2DM patients who were on $40 \mathrm{mg}$ statin was higher as compared to T2DM patients who were on $20 \mathrm{mg}$ statin therapy (Table 7). A study done by Auer et $\mathrm{al}^{46}$ showed as the doses of statins administered for patients increase muscle-related side effects will become more prevalent. Serum mean values of CK-MB isotype among T2DM who were on metformin + statin, insulin + statin and combined hypoglycemic drugs were not significantly different (Table 8).

Lactate dehydrogenase (LDH) is a cytoplasmic enzyme present in essentially all major organ systems. Ingestion of certain drugs, toxin and chemical poisons are among the major factors for LDH release to extracellular space. ${ }^{47}$ Elevated serum LDH could act as a biomarker for diagnosis of drug-induced hepatic and muscular injury. Previously published works done by Tokinaga et $\mathrm{al}^{20}$ and Noor et $\mathrm{al}^{48}$ showed that statin therapy is responsible for moderate-to-severe elevation of serum $\mathrm{LDH}^{20}$, The result of our study revealed that the mean serum level of LDH among T2DM patients who were on statin therapy for 14 days 6 months and $>18$ months showed non-significant elevation as compared to T2DM patients who were on statin for 618 months and not on statin therapy (Table 3). Previously published works done by Tokinaga et $\mathrm{al}^{20}$ and Noor et $\mathrm{al}^{48}$ reported that statin drugs can increase serum LDH through disruption of cellular integrity, especially when it has been taken together with other drugs. As independent $t$-test showed, there was moderate elevation of mean serum LDH level among T2DM patients who were on statin therapy compared to T2DM patients who were not on statin therapy (Table 6). In addition, mean serum values of LDH among T2DM patients who were on $40 \mathrm{mg}$ statin therapy were higher than LDH among T2DM patients who were on $20 \mathrm{mg}$ statin therapy (Table 8). This result was supported by a case report done by Dragos et $\mathrm{al}^{35}$ that showed elevation of LDH in T2DM patients who were on statin therapy. ${ }^{35}$ Another case report described by Unnikrishnan and Satish ${ }^{49}$ indicated that taking atorvastatin for several years elevated serum $\mathrm{CK}$ and $\mathrm{LDH}$.

\section{Conclusion}

Statin drugs were found to have the effect of lowering lipid profiles (total cholesterol, LDL- cholesterol and TAG) in T2DM patients and the effects were found to be prominent in T2DM patients who were on statin drugs for more than 18 months. In addition, $40 \mathrm{mg}$ statin dose produced a better effect on lipid profile as compared to $20 \mathrm{mg}$ statin dose. In this study, insulin + statin showed better results in decreasing lipid profile levels. Moderate elevation of liver enzymes, CK-MB and LDH were observed among T2DM patients who were on statin therapy as compared to T2DM patients who were not on statin therapy. The mean values of ALT and AST were found within normal reference range in all study groups. ALP was higher than normal values among all study groups. According to this study statin did not show significant toxicity to liver and muscle.

\section{Data Sharing Statement}

All necessary data and materials related to the article are included in the manuscript.

\section{Acknowledgments}

We would like to express our deepest gratitude to Haramaya University for providing the corresponding author with sponsorship and opportunity to conduct this research and Addis Ababa University for funding for this research project. We are extremely grateful to the Department of Medical Biochemistry, Addis Ababa University, for its indispensable support and coordination in the smooth running of this project. We would like to extend our acknowledgement to the study participants for their cooperation and willingness.

\section{Author Contributions}

All authors contributed to data analysis, drafting or revising the article, gave final approval of the version to be published, and agree to be accountable for all aspects of the work.

\section{Funding}

The research was funded by Addis Ababa University. The funder had no role in designing of the study, collection, analysis and interpretation of the data or in the writing of the manuscript.

\section{Disclosure}

The authors report no conflicts of interest in this work. 


\section{References}

1. Borle AL, Chhari N, Gupta G, Bathma V. Study of prevalence and pattern of dyslipidaemia in type 2 diabetes mellitus patients attending rural health training centre of medical college in Bhopal, Madhya Pradesh. Int J Community Med Public Health. 2017;3(1):140-144.

2. Mistire WG. Diabetes mellitus and associated diseases from Ethiopian perspective: systematic review. Ethiop $J$ Health Dev. 2013;27(3):249-253.

3. Krikorian A. Standards of medical care in diabetics. $J$ Am Diabet Assoc. 2016;39(Suppl. 1):S1-S2.

4. Kawanami D, Matoba K, Utsunomiya K. Dyslipidemia in diabetic nephropathy. Ren Replace Ther. 2016;2:16. doi:10.1186/s41100-0160028-0

5. Wu L, Parhofer KG. Diabetic dyslipidemia. Metab Clin Exp. 2014;63:1469-1514. doi:10.1016/j.metabol.2014.08.010

6. Mooradian AD. Dyslipidemia in type 2 diabetes mellitus. Nat Clin Pract Endocrinol Metab. 2009;5(3):150-159. doi:10.1038/ ncpendmet1066

7. Catapano AL, Graham I, De Backer G, et al. 2016 ESC/EAS Guidelines for the Management of Dyslipidaemias. Eur Heart J. 2016;37(39):2999-3058. doi:10.1093/eurheartj/ehw272

8. Basak RC, Chatterjee M, Sarma P. An overview on management of diabetic dyslipidemia. J Diabetes Endocrinol. 2013;4(3):27-36.

9. Gencer B, Mach F. Review of the 2016 European dyslipidaemia guidelines. Cardiovasc Med. 2016;19(11):283-287.

10. Rutishauser $\mathbf{J}$. The role of statins in clinical medicine-LDL-cholesterol lowering and beyond. Swiss Med Wkly. 2006;136(3/4):41-49.

11. Collins R, Reith C, Emberson J, et al. Interpretation of the evidence for the efficacy and safety of statin therapy. Lancet. 2016;388 (10059):2532-2561. doi:10.1016/S0140-6736(16)31357-5

12. Hennessy E, Adams C, Reen FJ, O'Gara F. Statins as next generation anti- microbials: is there potential for repurposing? Antimicrob Agents Chemother. 2016;AAC-00192.

13. Joo SJ. Anti-inflammatory effects of statins beyond cholesterol lowering. Korean Circ J. 2012;42(9):592-594. doi:10.4070/ kcj.2012.42.9.592

14. Bitztur R, Cohen H, Kamari Y, Harats D. Intolerance to statins: mechanisms and management. Diabetes Care. 2013;36(2):S325S330. doi: $10.2337 / \mathrm{dcS} 13-2038$

15. Russo MW, Hoofnagle JH, Gu J, et al. Spectrum of statin hepatotoxicity: experience of the drug-induced liver injury network. Hepatology. 2014;60(1):679-686. doi:10.1002/hep.27157

16. Stroes ES, Thompson PD, Corsini A, et al. Statin-associated muscle symptoms: impact on statin therapy-European Atherosclerosis Society Consensus Panel statement on assessment, aetiology and management. Eur Heart J. 2015;36(17):1012-1022. doi:10.1093/eurheartj/ehv043

17. Maji D, Shaikh S, Solanki D, Gaurav K. Safety of statins. Lancet. 2013;17(4):636-646. doi:10.4103/2230-8210.113754

18. Ambapkar S, Shetty N, Dwivedy A. Statin-Induced Rhabdomyolysis. J Associ Physi India. 2016;64:93-94.

19. Pedro-Botet J, Millán Núñez-Cortés J, Chillarón JJ, Flores-le Roux JA, Rius J. Severity of statin-induced adverse effects on muscle and associated conditions: data from the DAMA study. Expert Opin Drug Saf. 2016;15(12):1583-1587. doi:10.1080/14740338.2016.1238068

20. Tokinaga K, Oeda T, Suzuki Y, Matsushima Y. HMG-CoA Reductase Inhibitors (statins) might cause high elevation of creatine phosphokinase (CPK) in patients with unnoticed hypothyroidism. Endo $J$. 2006;53(3):401-405. doi:10.1507/endocrj.K04-144

21. Moghadam-kia S, Oddis CV, Aggarwal R. Approach to asymptomatic creatine kinase elevation. Cleve Clin J Med. 2016;83(1):3742. doi:10.3949/ccjm.83a.14120

22. Faloppi L, Bianconi M, Memeo R, et al. Lactate dehydrogenase in hepatocellular carcinoma: something old, something new. Biomed Res Int. 2016;2016. doi:10.1155/2016/7196280
23. Miao P, Sheng S, Sun X, Liu J, Huang G. Lactate dehydrogenase A in cancer: a promising target for diagnosis and therapy. IUBMB Life. 2013;65(11):904-910. doi:10.1002/iub.1216

24. Imai K, Watari S, Sakazume T, Mitsuyama S. Clinical chemistry and immunoassay testing supporting the individual healthy life. Hitachi Rev. 2008;57:1-7.

25. Balgi V, Harshavardan L, Sahna E, Thomas SK. Pattern of lipid profile abnormality in subjects with prediabetes. Int J Sci Study. 2017;4(11):150-153.

26. Trentman TL, Avey SG, Ramakrishna H. Current and emerging treatments for hypercholesterolemia: a focus on statins and proprotein convertase subtilisin/kexin Type 9 inhibitors for perioperative clinicians. $J$ Anaesthesiol Clin Pharmacol. 2016;32:4405. doi:10.4103/09709185.194773

27. Bonetti PO, Lerman LO, Napoli C, Lerman A. Statin effects beyond lipid lowering - are they clinically relevant? Eur Heart J. 2003;24 (3):225-248. doi:10.1016/S0195-668X(02)00419-0

28. Stancu C, Sima A. Statins: mechanism of action and effects. $J$ Cell Mol Med. 2001;5(4):378-387. doi:10.1111/jcmm.2001.5.issue-4

29. Takata K, Psaltis PJ, Nicholls SJ. Investigate the long term legacy of statin therapy. J Thorac Dis. 2017;9(4):936-939. doi:10.21037/jtd.2017.03.10

30. Ford I, Murray H, McCowan C, Packard CJ. Long term safety and efficacy of lowering LDL cholesterol with statin therapy: 20-Year Follow-Up of West of Scotland Coronary Prevention study. Circulation. 2016;115:1073-1080.

31. Lu Y, Cheng Z, Zhao Y, et al. Efficacy and safety of long term treatment with statin for coronary heart disease: a Bayesian network meta-analysis. Atherosclerosis. 2016;254:215-227. doi:10.1016/j. atherosclerosis.2016.10.025

32. Han H, He Y, Hu J, Lau R, Lee H, Ji C. Disrupted ER-to-golgi trafficking underlies anti-HIV drugs and alcohol-induced cellular stress and hepatic injury. Hepatol Commun. 2017;1(2):122-139. doi:10.1002/hep4.1030

33. Kullak-Ublick GA, Andrade RJ, Merz M, et al. Drug induced liver injury: recent advances in diagnosis and risk assessment. Gut. 2017;2016:1154-1164.

34. Marrone G, Vaccaro FG, Biolato M, et al. Drug-induced liver injury: the diagnosis is not easy but always to keep in mind. Eur Rev Med Pharmacol Sci. 2017;21(1 Suppl):122-134.

35. Dragos D, Pruteanu D, Constantin R. High-dose statin associated with rhabdomyolysis, acute kidney injury, cholestatic liver injury, and thrombocytopenia. J Nephrol Kidney Dis. 2017;1(1):1001. doi:10.36876/smjnkd

36. Thapar M, Russo MW, Bonkovsky HL. Statins and liver injury. Gastroenterol Hepatol (N Y). 2013;9(9):605.

37. Gao Y, Wang Y, Wu NQ, et al. Statins use and the risk of liver dysfunction: a Chinese cohort study in real world clinical practice. IJC Metab Endocr. 2017;16:16-20. doi:10.1016/j.ijcme.2017.06.003

38. Björnsson ES. Hepatotoxicity by drugs: the most common implicated agents. Int J Mol Sci. 2016;17(2):224. doi:10.3390/ijms17020224

39. Clarke AT, Johnson PC, Hall GC, Ford I, Mills PR. High dose atorvastatin associated with increased risk of significant hepatotoxicity in comparison to simvastatin in UK GPRD cohort. PLoS One. 2016;11(3):e0151587. doi:10.1371/journal.pone.0151587

40. Ortega-Alonso A, Stephens C, Lucena MI, Andrade RJ. Case characterization, clinical features and risk factors in drug-induced liver injury. Int J Mol Sci. 2016;17(5):714. doi:10.3390/ijms17050714

41. Egholm G, Pareek M.Drug-induced rhabdomyolysis with elevated cardiac troponin T. Case Rep Med.2015;2015:1-3. doi:10.1155/2015/270204

42. Parker BA, Capizzi JA, Grimaldi AS, et al. Effect of statins on skeletal muscle function. Circulation. 2013;127:96-103. doi:10.11 61/CIRCULATIONAHA.112.136101

43. Soko N, Dandara C, Ramesar R, Kadzirange G, Masimirembwa C. Pharmacokinetics of rosuvastatin in 30 healthy Zimbabwean individuals of African ancestry. Br J Clin Pharmacol. 2016;82:326-328. doi: $10.1111 /$ bcp. 12915 
44. Calza L, Magistrelli E, Colangeli V, et al. Significant association between statin-associated myalgia and vitamin $\mathrm{D}$ deficiency among treated HIV-infected patients. AIDS. 2017;31:681-688. doi:10.1097/ QAD.0000000000001397

45. Holder K. Myalgias and myopathies: drug-induced myalgias and myopathies. FP Essent. 2016;440:23-27.

46. Auer J, Sinzinger H, Franklin B, Berent R. Muscle- and skeletalrelated side- effects of statins: tip of the iceberg? Eur J Prev Cardiol. 2016;23(1):88-110. doi:10.1177/2047487314550804

47. Mohammed NB, Elias SE. Assessment of gamma glutamyl transferase and lactate dehydrogenase as biochemical markers of preeclampsia severity in sudanese pregnant women'sin Khartoum State. Savant J Med Sci. 2016;2(9):177-183.
48. Noor M, Waheed A, Muhammad I, Bakhtiar S. Is co q10 really valuable in shielding statin induced myopathy-an exploration? Pakistan Armed Forces Med J. 2016;66(4):525-529.

49. Unnikrishnan D, Satish B. Exertion induced rhabdomyolysis in patients on statin therapy. Nephrol Dial Transplant. 2005;20(1):24. doi:10.1093/ndt/gfh578

Diabetes, Metabolic Syndrome and Obesity: Targets and Therapy

\section{Publish your work in this journal}

Diabetes, Metabolic Syndrome and Obesity: Targets and Therapy is an international, peer-reviewed open-access journal committed to the rapid publication of the latest laboratory and clinical findings in the fields of diabetes, metabolic syndrome and obesity research. Original research, review, case reports, hypothesis formation, expert opinion and commentaries are all considered for publication. The manuscript management system is completely online and includes a very quick and fair peer-review system, which is all easy to use. Visit http://www.dovepress.com/testimonials.php to read real quotes from published authors. 